\title{
表面活性剂对羟基磷灰石纤维形貌的影响
}

$$
\text { 齐美丽 }{ }^{1,2,3} \text {, 元 佳 }{ }^{1,2} \text {, 肖桂勇 }{ }^{1,2,3} \text {, 吕宇鹏 } 1,2,3
$$

(1. 山东大学 材料液固结构演变与加工教育部重点实验室, 济南 250061; 2. 山东大学 材料科学与工程学院, 济 南 250061; 3. 山东大学 苏州研究院, 苏州 215123)

摘 要: 以 $\mathrm{Ca}\left(\mathrm{NO}_{3}\right)_{2} \cdot 4 \mathrm{H}_{2} \mathrm{O}$ 和 $\left(\mathrm{NH}_{4}\right)_{2} \mathrm{HPO}_{4}$ 水溶液为前驱体, 采用水热均相沉淀法制备了结晶度较高的羟基磷灰石纤 维，研究了表面活性剂十六烷基三甲基溴化胺(CTAB)、十二烷基硫酸钠(SDS)和聚乙二醇(PEG)及其含量对产物形 貌和相组成的影响。结果表明, 采用这三种表面活性剂制备的产物都是羟基磷灰石, 部分样品含有少量碳酸钙杂 质。加入 CTAB 和 SDS 均会对纤维的生长起到抑制作用, 得到纤维与球形团聚体并存的产物, 而 PEG 的加入在一 定程度上促进了纤维的生长。

关 键 词: 羟基磷灰石纤维; 表面活性剂; 水热均相沉淀; 形貌

中图分类号: TB321 文献标识码: A

\section{Effect of Surfactants on the Morphology of Hydroxyapatite Fibers}

\author{
QI Mei-Li ${ }^{1,2,3}$, QI Jia ${ }^{1,2}$, XIAO Gui-Yong ${ }^{1,2,3}$, LV Yu-Peng ${ }^{1,2,3}$
}

(1. Key Laboratory for Liquid-Solid Structural Evolution and Processing of Materials, Ministry of Education, Shandong University, Ji'nan, 250061, China; 2. School of Materials Science and Engineering, Shandong University, Ji'nan, 250061, China; 3. Shandong University Suzhou Institute, Suzhou 215123, China)

\begin{abstract}
Hydroxyapatite fiber with high crystallinity was successfully fabricated by hydrothermal homogeneous precipitation synthesis with $\mathrm{Ca}\left(\mathrm{NO}_{3}\right)_{2} \cdot 4 \mathrm{H}_{2} \mathrm{O}$ and $\left(\mathrm{NH}_{4}\right)_{2} \mathrm{HPO}_{4}$ as precursors. Effects of the surfactants include cetyltrimethylammonium bromide (CTAB), sodium dodecyl sulfate (SDS) and polyethylene glycol (PEG), and different amounts of them on the morphology and phase composition of the obtained products were investigated. Results show that the products obtained by using the three surfactants are all hydroxyapatite with minor $\mathrm{CaCO}_{3}$ in some samples. The addition of both CTAB and SDS inhibits the growth of the fibers, leading to the coexistence of fibers and spherical aggregates. However, the addition of PEG can promote the growth of fibers to some extent.
\end{abstract}

Key words: hydroxyapatite fibers; surfactants; hydrothermal homogeneous precipitation; morphology

羟基磷灰石(Hydroxyapatite, HA) 是组成人体 骨骼和牙齿主要的无机成分, 因具有优异的生物 相容性、骨传导性、生物活性及安全无毒性等诸多 优点, 被广泛应用于硬组织缺损的修复与再生以
及载药释药等领域 ${ }^{[1-3]}$ 。然而, 脆性大这一缺陷限 制了其在承重修复体中的应用。为改善 HA 的脆性, 人们进行了多方面的研究, 如利用碳纳米管、氧化 锆纤维、氧化铝纤维等作为增强相 ${ }^{[4-7]}$ 。某些纤维

收稿日期: 2015-10-10; 收到修改稿日期：2015-12-29

基金项目：山东大学基本科研业务费专项基金(2015JC018); 江苏省青年科学基金(BK20140412); 山东省优秀中青年科学家 科研奖励基金(BS2013CL030)

Special Fund of Shandong University Basic Research (2015JC018); Jiangsu Province Science Foundation for Youths (BK20140412); Shangdong Province Young and Middle-Aged Scientists Research Awards Fund (BS2013CL030)

作者简介: 齐美丽(1988-), 女，博士研究生. E-mail: beauty0507@163.com

通讯作者：吕宇鹏，教授. E-mail: biosdu@sdu.edu.cn 
尽管在形貌上能够达到 Stanton-Pott 无害标准, 但 复合植入体的生物活性和生物相容性受到了不良 影响 ${ }^{[8]}$ 。因此, 开发柔韧性好且均匀连续的羟基磷灰 石纤维(hydroxyapatite fiber, HAF)将为改善 HA 基生 物材料的力学性能提供新的途径。

尽管通过固相法 ${ }^{[9]}$ 、熔盐法 ${ }^{[10]}$ 、水热法 ${ }^{[11]}$ 、静 电纺丝法 ${ }^{[12]}$ 等方法可以制备出 HA 晶须或短纤维, 但产物对制备条件要求较高, 并且结晶度和㓞性也 较差。水热均相沉淀法是一种制备高长径比、高结 晶度 HAF 的简便方法。其原理是通过酰胺类沉淀剂 随温度的升高和时间的延长不断水解从而缓慢调节 体系的 $\mathrm{pH}$ 这一性质, 控制溶液中 $\mathrm{OH}^{-}$的释放速度使 之 $\mathrm{HA}$ 的合成同步, 使得晶体在生长过程中得到均 匀的形貌, 诱导 $\mathrm{HA}$ 生长成纤维状。目前, 利用这一 方法制备出 HA 晶须已有报道 ${ }^{[13-14]}$, 但组分和形态 的可控制备仍需进一步研究。而采用表面活性剂等 多用于水热及化学沉淀等方法的 HA 制备 ${ }^{[15-18]}$ 。但 尚未见采用表面活性剂调控制备 HAF 尺寸及形貌 的报道。

本研究旨在研究阳离子型表面活性剂十六烷基 三甲基溴化胺(CTAB)、阴离子型表面活性剂十二烷 基硫酸钠(SDS)和非离子型表面活性剂聚乙二醇 (PEG)对水热均相沉淀法合成 HAF 的影响, 在反应 时间与反应温度为定值的情况下, 分别观察采用不 同的表面活性剂含量所合成的 HAF 的形貌特点, 以 期为高长径比高柔韧性 HAF 的制备提供实验依据。

\section{1 实验方法}

\section{1 实验材料及水热合成}

实验所用材料 $\mathrm{Ca}\left(\mathrm{NO}_{3}\right)_{2} \cdot 4 \mathrm{H}_{2} \mathrm{O} 、\left(\mathrm{NH}_{4}\right)_{2} \mathrm{HPO}_{4}$ 、 尿素、硝酸、CTAB、SDS 和 PEG 统一购自于国药 集团化学试剂有限公司, 分析纯。

按照 $\mathrm{Ca} / \mathrm{P}=1.8$ 配制 $\mathrm{Ca}\left(\mathrm{NO}_{3}\right)_{2} \cdot 4 \mathrm{H}_{2} \mathrm{O}$ 和 $\left(\mathrm{NH}_{4}\right)_{2}$ $\mathrm{HPO}_{4}$ 溶液后与 $1 \mathrm{~mol} / \mathrm{L}$ 的尿素溶液混合, 分别加入 CTAB、SDS 和 PEG 并用硝酸调节溶液的 $\mathrm{pH}$ 至 3.5 。 试验中 CTAB 的加入量分别为 $1.84 、 3.9$ 和 $9.2 \mathrm{mmol} / \mathrm{L}$, SDS 的加入量分别为 $0.435 、 0.87$ 和 $4.35 \mathrm{mmol} / \mathrm{L}$, PEG 分别按照 $n\left(\mathrm{Ca}^{2+}\right): n\left(\mathrm{PO}_{4}{ }^{3-}\right): n(\mathrm{SDS})=10.8: 6: 1$ 、 10.8:6:2 和 10.8:6:10 添加。将上述前驱体溶液倒 入聚四氟乙烯内祄的不锈钢反应釜中, 控制填充 度为 $80 \%$, 反应温度为 $180^{\circ} \mathrm{C}$, 保温时间为 $10 \mathrm{~h}$ 。 然后将产物离心、去离子水清洗 1 次、无水乙醇 清洗 2 次后放入 $80^{\circ} \mathrm{C}$ 鼓风干燥箱中干燥 $3 \mathrm{~h}$, 得到 最终产物。

\section{2 表征}

利用日本产的 Rigaku D/max- $\gamma$ B 型 X 射线衍射 仪(XRD)对产物的相组成进行检测分析, 测试条件: 铜靶 $(\mathrm{Cu}-\mathrm{K} \alpha)$; 管电压和管电流分别 $40 \mathrm{kV}$ 和 $100 \mathrm{~mA}$, 扫描速度 $4 \% \mathrm{~min}$, 扫描范围 $10^{\circ} \sim 60^{\circ}$ 。利用 SU-70 型 日立热场发射扫描电镜(FE-SEM)对产物的表面形貌 进行分析, 加速电压和加速电流分别设定为 $5 \mathrm{kV}$ 和 $10 \mu \mathrm{A}$ ，测试前进行喷金处理。

\section{2 结果与讨论}

\section{1 物相分析}

图 1 是未添加任何表面活性剂与分别添加不同 量的 CTAB、SDS 和 PEG 后制备产物的 XRD 图谱 及 HA 的标准图谱(JCPDS 09-0432)。由图可知, HA 峰形较为尖锐, 说明其结晶性较高(图 1(a))。添加 三种表面活性剂前后得到的产物衍射峰的峰形基 本一致, 但相对强度稍有差异, 这与其他文献报道 相似 ${ }^{[9,19-20]}$, 推测是由晶体的择优取向不同导致的。 添加 SDS 后产物的结晶度略差(图 1(c)), 而添加 PEG 后在 $29.5^{\circ}$ 左右出现了 $\mathrm{CaCO}_{3}$ 的一个较强峰(图 $1(\mathrm{~d})) \circ \mathrm{CO}_{3}{ }^{2-}$ 的出现一方面是由于高压釜封装时需要 留余一部分空间, 空气中的 $\mathrm{CO}_{2}$ 进入, 另一方面尿 素在高温分解产生 $\mathrm{CO}_{2}$ 。大部分 $\mathrm{CO}_{2}$ 从反应体系中 释放出来, 但仍有一小部分溶于水中。溶于水中的 $\mathrm{CO}_{3}{ }^{2-}$ 很容易取代 $\mathrm{HA}$ 晶体中的部分阴离子或与 $\mathrm{Ca}^{2+}$ 的结合生成 $\mathrm{CaCO}_{3}$, 也是产物缺钙的原因之一。另 外, SDS 加入量为 $4.35 \mathrm{mmol} / \mathrm{L}$ 时出现了一个较明显 的非晶包, 推测可能是由于部分 $\mathrm{Ca}-\mathrm{P}$ 相未充分转变 引起。由图可知, 所选取的这三种有代表性的表面 活性剂对产物的物相影响不明显。

\section{2 微观形貌分析}

\subsubsection{CTAB 对产物形貌的影响}

图 2 是添加不同量的 CTAB 后制备的产物 SEM 照片, 图 2(a)为无添加剂的原始试样形貌, $(\mathrm{b} \sim \mathrm{d})$ 分 别为添加 1.84、3.9 和 $9.2 \mathrm{mmol} / \mathrm{L} \mathrm{CTAB}$ 后得到的产 物形貌。从图中可以看出, 加入 $1.84 \mathrm{mmol} / \mathrm{L}$ 的 CTAB 反应得到的 HAF 与原始试样均是从一个团聚 体出发生长, 微观形貌呈带状, 从图 2(a,b)中的插图 可以看出纤维表面光滑平整, 呈明显弯曲形态。随 着添加量的增加, 产物中出现微球(图 2(c))。添加剂 的量继续增加后, 产物中球状物质增加, 同时部分 尺寸较大的片状物质从形核点处脱落, 产物形貌不 均匀(图 2(d))。 

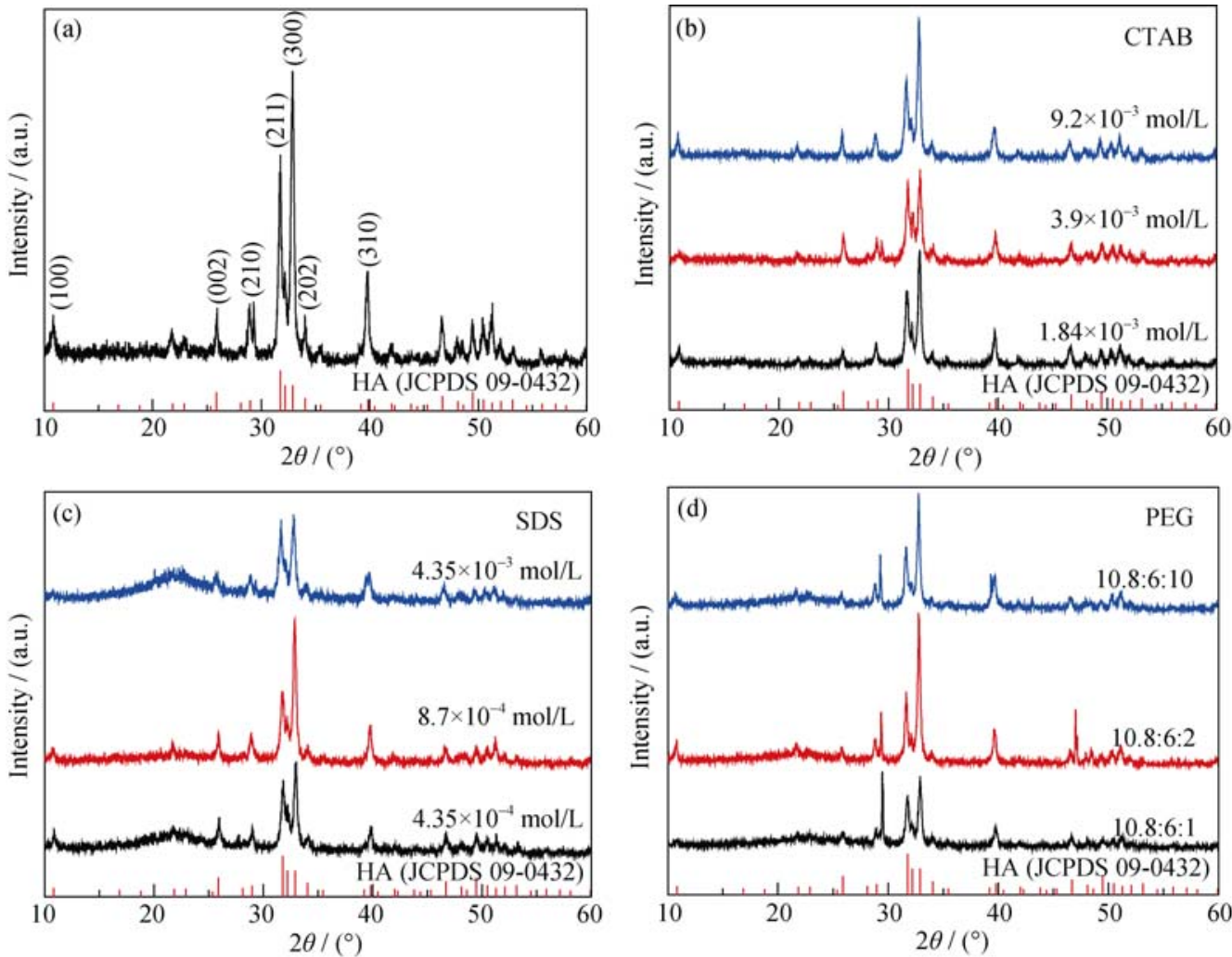

图 1 未添加及分别添加不同量的表面活性剂后制备产物的 XRD 图谱

Fig. 1 XRD patterns of the products synthesized without (a) and with the addition of different amounts of surfactants (b-d)
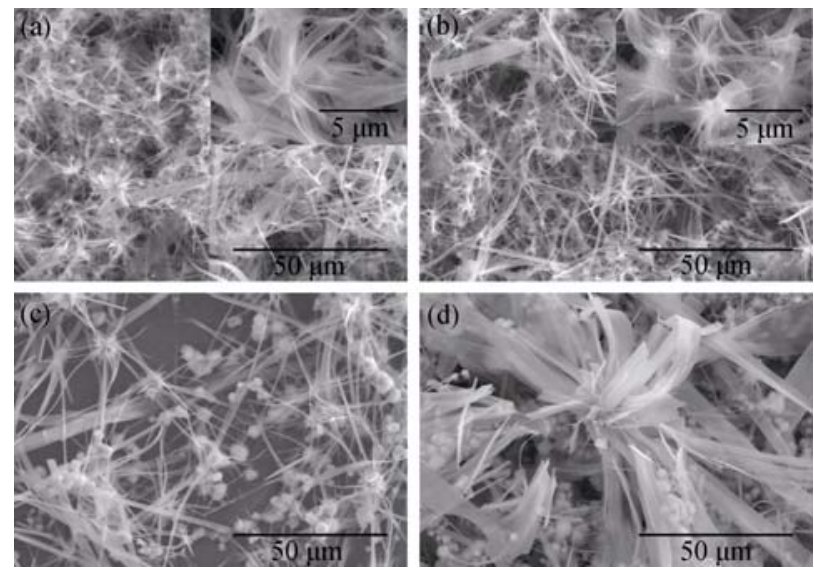

图 2 未添加及分别添加不同量的 CTAB 后制备的产物 SEM 照片

Fig. 2 SEM images of the products with addition of different amounts of CTAB

(a) $0 \mathrm{mmol} / \mathrm{L}$; (b) $1.84 \mathrm{mmol} / \mathrm{L}$; (c) $3.9 \mathrm{mmol} / \mathrm{L}$; (d) $9.2 \mathrm{mmol} / \mathrm{L}$

选用尿素为 $\mathrm{pH}$ 调节剂控制体系的 $\mathrm{pH}$ 比较温 和，水热反应前用硝酸将钙源和磷源混合后得到的 磷酸氢钙沉淀溶解, 然后加热到 $180^{\circ} \mathrm{C}$, 利用尿素 可在 $80^{\circ} \mathrm{C}$ 以上缓慢水解释放 $\mathrm{OH}^{-}$这一原理, 使溶液 的 $\mathrm{pH}$ 不断升高, 避免了 $\mathrm{OH}^{-}$浓度不均衡的状态, 从 而得到具有均匀形貌的 $\mathrm{HAF}$ 。其水解方程式为:

$\mathrm{CO}\left(\mathrm{NH}_{2}\right)_{2}+3 \mathrm{H}_{2} \mathrm{O} \rightarrow 2 \mathrm{NH}_{4}{ }^{+}+2 \mathrm{OH}^{-}+\mathrm{CO}_{2}$
$\mathrm{CTAB}$ 是阳离子表面活性剂的典型代表，室温 时, 其临界胶束浓度为 $0.92 \mathrm{mmol} / \mathrm{L}$ 。由上图可知, $\mathrm{CTAB}$ 的加入对 $\mathrm{HAF}$ 的生长起抑制作用，当 $\mathrm{CTAB}$ 的浓度大于 $3.9 \mathrm{mmol} / \mathrm{L}$ 时，产生显著抑制，出现微 球与纤维并存的现象, 产物形貌不均。本实验中, $\mathrm{CTAB}$ 对 $\mathrm{HA}$ 晶体生长的影响主要体现在 ${ }^{[21]}: \mathrm{PO}_{4}{ }^{3-}$ 离子粘附在胶束的表面，对于 $\mathrm{C}$ 面生长基元 Ca- $\mathrm{P}_{6} \mathrm{O}_{24}$ 来说, $\mathrm{CTAB}$ 与 $\mathrm{PO}_{4}{ }^{3-}$ 的作用将阻碍负离子 配位体生长基元 $\mathrm{Ca}-\mathrm{P}_{6} \mathrm{O}_{24}$ 的形成。 $\mathrm{CTAB}$ 可能吸附 在 $\mathrm{Ca}-\mathrm{P}_{6} \mathrm{O}_{24}$ 基元上阻碍其进入 $\mathrm{HA}$ 晶体，从而导致 $\mathrm{HA}$ 晶体在 $c$ 轴方向的生长速度变慢, 从宏观上看, HA 长径比变小。

\subsubsection{SDS 对产物形貌的影响}

图 3 是添加不同量的 SDS 后制备的产物 $\mathrm{SEM}$ 照片，图 3(a c ) 分别为添加 0.435、0.87 和 $4.35 \mathrm{mmol} / \mathrm{L}$ 的 SDS 后得到的产物形貌。从图中 可以看出, 加入 $0.435 \mathrm{mmol} / \mathrm{L}$ 的 SDS 反应得到的 $\operatorname{HAF}$ (图 3(a)) 与原始试样微观形貌(图 2(a)) 相似, 团聚较严重。随 SDS 浓度的增加, 也是呈现出微 球与片状晶体并存的现象(图 3(b)); 随 SDS 的添 加量继续增加, 球状形貌消失, 得到不规则的花 瓣状 $\mathrm{HA}$ (图 3(c))。 

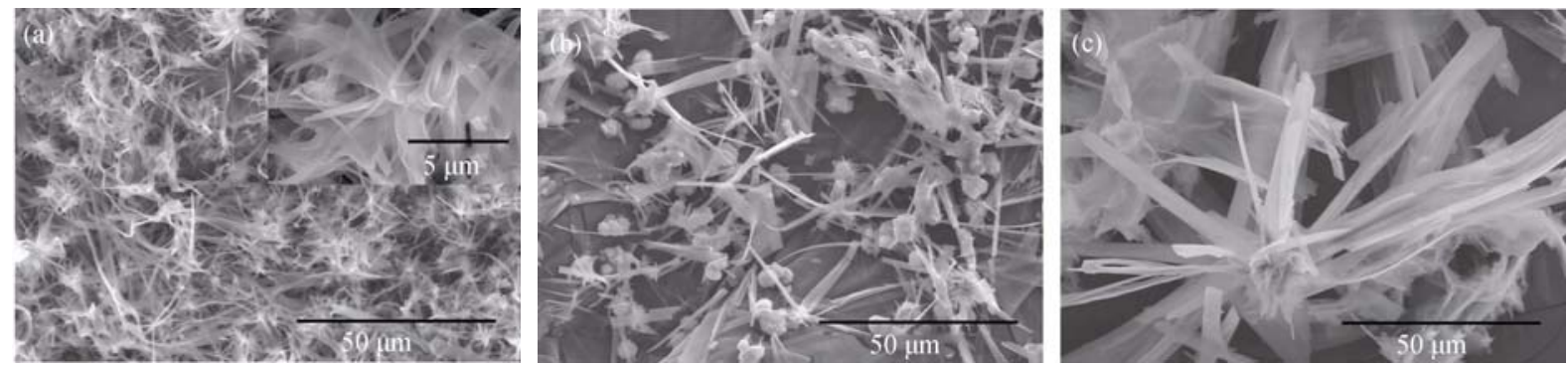

图 3 添加不同量的 SDS 后制备的产物 SEM 照片

Fig. 3 SEM images of the products with addition of different amounts of SDS (a) $0.435 \mathrm{mmol} / \mathrm{L}$; (b) $0.87 \mathrm{mmol} / \mathrm{L}$; (c) $4.35 \mathrm{mmol} / \mathrm{L}$

SDS 是阴离子表面活性剂的典型代表, 其阴离 子长链可以吸附 $\mathrm{Ca}^{2+}$, 可与 $\mathrm{OH}^{-}$竞争吸附 $\mathrm{Ca}^{2+}$ 位。 $\mathrm{SDS}$ 的临界胶束浓度为 $8.7 \mathrm{mmol} / \mathrm{L}\left(40^{\circ} \mathrm{C}\right)$, 随温度 的升高, 临界胶束浓度增大。由上图可知, SDS 的加 入也对 HAF 的生长起抑制作用, 当 SDS 的浓度大 于 $0.87 \mathrm{mmol} / \mathrm{L}$ 时, 产生显著抑制, 得到 $\mathrm{HA}$ 形貌由微 球与片状晶体并存变为花瓣状。反应过程中经历了从 磷酸氢钻向亚稳态磷酸八钲 $\left(\mathrm{OCP}, \mathrm{Ca}_{8} \mathrm{H}_{2}\left(\mathrm{PO}_{4}\right)_{6} \cdot 5 \mathrm{H}_{2} \mathrm{O}\right)$ 转变, 然后 $\mathrm{OCP}$ 脱水沿 $\mathrm{b} 、 \mathrm{c}$ 轴方向裂开成条状转 变为 HA 晶体的这一过程 ${ }^{[22]}$ 。OCP 在转变成 HA 后, 原来易于吸附富含有羧基的有机物的(100)面仍然 很可能被有机物优先吸附 ${ }^{[23]}$, 因而带负电的基团 SDS 能够吸附在 HA 的(100)面。这种吸附作用能阻 碍 $\mathrm{HA}(100)$ 面的生长, 使(100)面扩大成为显露面, 从而使 HA 大多形成片状形貌 ${ }^{[21]}$, 这一点可以从图 3 中得到验证, 加入表面活性剂 SDS 后得到了片状
的 HA 晶体。

\subsubsection{PEG 对产物形貌的影响}

由于 PEG 是非离子型表面活性剂, 无法做成胶 束, 本研究中 PEG 的添加量依据钙磷与 PEG 的摩尔 比确定, 产物形貌如图 4 所示。图 4(a c) 分别为 $n\left(\mathrm{Ca}^{2+}\right): n\left(\mathrm{PO}_{4}{ }^{3-}\right): n(\mathrm{PEG})=10.8: 6: 1,10.8: 6: 2$ 和 10.8:6:10。 与图 2(a)相比, 可以明显看出, 加入 PEG 后产物的 团聚体变大, 纤维长大。

在本实验中, PEG 在一定程度上促进了 $\mathrm{HAF}$ 的 生长。有研究表明: $\mathrm{Ca}^{2+}$ 与 $\mathrm{PEG}$ 之间存在相互作用, 降低了 $\mathrm{Ca}^{2+}$ 的迁移速度, 从而降低了电导率, 这种 相互作用，可能是 PEG 对 HA 晶体成核和生长造成 影响的原因之一 ${ }^{[24]}$ 。PEG 的存在，可以修饰或控制 $\mathrm{HA}$ 晶体的表面，在其成核的过程中起到分散作用， 促进晶体的生长。
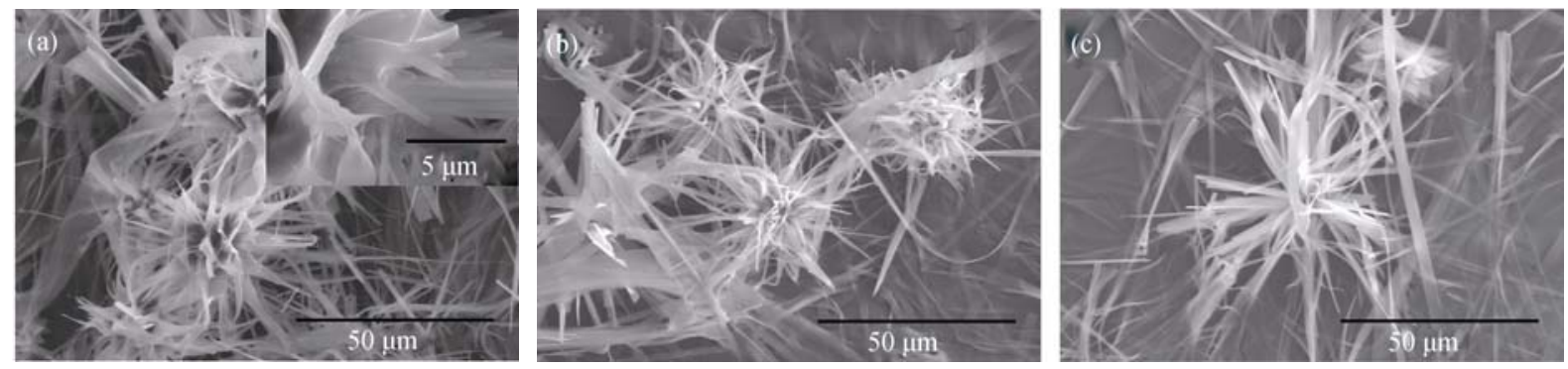

图 4 添加不同量的 PEG 后制备的产物 SEM 照片

Fig. 4 SEM images of the products with addition of different amounts of PEG (a) $n\left(\mathrm{Ca}^{2+}\right): n\left(\mathrm{PO}_{4}{ }^{3-}\right): n(\mathrm{PEG})=10.8: 6: 1 ;$ (b) $n\left(\mathrm{Ca}^{2+}\right): n\left(\mathrm{PO}_{4}{ }^{3-}\right): n(\mathrm{PEG})=10.8: 6: 2 ;$ (c) $n\left(\mathrm{Ca}^{2+}\right): n\left(\mathrm{PO}_{4}{ }^{3-}\right): n(\mathrm{PEG})=10.8: 6: 10$

\section{3 结论}

1) 采用水热均相沉淀法成功合成了结晶度较 高的 HAF, 纤维从一个团聚体出发生长, 微观形貌 呈带状，且表面光滑平整，弯曲性较好;

2) CTAB 和 SDS 的添加均会阻碍 HA 长成纤维 状, 得到的产物中出现球形团聚体; 而 PEG 的加入 在一定程度上促进了 HAF 的生长。

\section{参考文献:}

[1] CHEN C W, OAKES C S, BYRAPPA K, et al. Synthesis, characterization, and dispersion properties of hydroxyapatite prepared by mechanochemical-hydrothermal methods. Journal of Materials Chemistry, 2004, 14(15): 2425-2432.

[2] VALLET-REGI M, GONZALEZ-CALBET J M. Calcium phosphates as substitution of bone tissues. Progress in Solid State 
Chemistry, 2004, 32(1/2): 1-31.

[3] MA Y J, HAO L J, DU S L, et al. Synthesis of hydroxyapatite microspheres by hydrothermal method under the control of sodium citrate. Journal of Inorganic Materials, 2014, 29(3): 284-288.

[4] LAHIRI D, GHOSH S, AGARWAL A. Carbon nanotube reinforced hydroxyapatite composite for orthopedic application: a review. Materials Science and Engineering C, 2012, 32(7): 1727-1758.

[5] AFZAL1 M A F, KESARWANI P, REDDY K M, et al. Functionally graded hydroxyapatite-alumina-zirconia biocomposite: synergy of toughness and biocompatibility. Materials Science and Engineering C, 2012, 32(5): 1164-1173.

[6] YANG C, GUO Y K, ZHANG M L. Thermal decomposition and mechanical properties of hydroxyapatite ceramic. Transactions of Nonferrous Metals Society of China, 2010, 20(2): 254-258.

[7] MITTAL M, NATH S K, PRAKASH S. Improvement in mechanical properties of plasma sprayed hydroxyapatite coatings by $\mathrm{Al}_{2} \mathrm{O}_{3}$ reinforcement. Materials Science and Engineering C. 2013, 33(5): 2838-2845.

[8] YOSHIMURA M, SUDA H, OKAMOTO K, et al. Hydrothermal synthesis of biocompatible whiskers. Journal of Materials Science, 1994, 29(13): 3399-3402

[9] OTA Y, IWASHITA T. Novel preparation method of hydroxyapatite fibers. Journal of the American Ceramic Society, 1998, 81(6): $1665-1668$.

[10] TAS A C. Molten salt synthesis of calcium hydroxyapatite whiskers. Journal of the American Ceramic Society, 2001, 84(2): 295-300.

[11] LI C H, LIU S G, LI G C, et al. Hydrothermal synthesis of large-sized hydroxyapatite whiskers regulated by glutamic acid in solutions with low supersaturation of precipitation. Advanced Powder Technology, 2011, 22(4): 537-543.

[12] FRANCO P Q, JOAO C F C, SILVA J C, et al. Electrospun hydroxyapatite fibers from a simple Sol-Gel system. Materials Letters, 2012, 67(1): 233-236.

[13] ZHANG H, DARVELL B W. Morphology and structural characteristics of hydroxyapatite whiskers: effect of the initial $\mathrm{Ca}$ con- centration, $\mathrm{Ca} / \mathrm{P}$ ratio and $\mathrm{pH}$. Acta Biomaterialia, 2011, 7(7): 2960-2968.

[14] HAO L J, YANG H, ZHAO N R, et al. Controlled growth of hydroxyapatite fibers precipitated by propionamide through hydrothermal synthesis. Powder Technology, 2014, 253: 172-177.

[15] WANG J, HUANG S P, HU K, et al. Effect of cetyltrimethylammonium bromide on morphology and porous structure of mesoporous hydroxyapatite. Transacfions of Nonferrous Metals Society of China, 2015, 25(2): 483-489.

[16] YAN L, LI Y D, DENG Z X, et al. Surfactant-assisted hydrothermal synthesis of hydroxyapatite nanorods. International Journal of Inorganic Materials, 2001, 3(7): 633-637.

[17] 高 珊. 表面活性剂对羟基磷灰石纳米线合成的影响研究. 济南: 山东大学硕士学位论文, 2012.

[18] GAO S, SUN K N, LI A M, et al. Synthesis and characterization of hydroxyapatite nanofiber by chemical precipitation method using surfactants. Materials Research Bulletin, 2013, 48(3): 1003-1006.

[19] ROEDER R K, CONVERSE G L, LENG H J, et al. Kinetic effects on hydroxyapatite whiskers synthesized by the chelate decomposition method. Journal of the American Ceramic Society, 2006, 89(7): 2096-2104.

[20] VISWANATH B, RAVISHANKAR N. Controlled synthesis of plate-shaped hydroxyapatite and implications for the morphology of the apatite phase in bone. Biomaterials, 2008, 29(36): 4855-4863.

[21] 张海斌. 羟基磷灰石晶粒/粒子的水热控制合成. 长沙：中南大 学博士学位论文, 2011 .

[22] IIJIMA M, KAMEMIZU H, WAKAMATSU N, et al. Effects of Ca addition on the formation of octacalcium phosphate and apatite in solution at pH 7.4 and at $37^{\circ} \mathrm{C}$. Journal of Crystal Growth, 1998, 193(1/2): $182-188$.

[23] BALASUNDARAM G, SATO M, WEBSTER T J. Using hydroxyapatite nanoparticles and decreased crystallinity to promote osteoblast adhesion similar to functionalizing with RGD. Biomaterials, 2006, 27(14): 2798-2805.

[24] 邱超凡. 球状羟基磷灰石的生物矿化合成研究. 福州: 福建师 范大学硕士学位论文, 2007. 